\title{
Las "estaciones de aprendizaje" como experiencia docente innovadora en la asignatura Antropología social, en el Grado en Criminología
}

\section{The "learning stations" as an innovative teaching experience in Social Anthropology subject, in the Degree in Criminology}

MARÍA MUÑIZ RIVAS

ORCID: https://orcid.org/0000-0002-6086-2580 Universidad de Sevilla

Departamento de Antropología Social mmrivas@us.es

Fecha de recepción: 31-05-2019

Fecha de aceptación: 13-06-2019

DOI: http://dx.doi.org/10.12795/9788447221912.033 Pp.: $759-780$ 
En la asignatura Antropología Social, de primer curso del Grado en Criminología, se desarrolló un Ciclo de Mejora en el Aula (CIMA) en el tema de la asignatura titulado "Organización social y política", ocupando el ciclo un total de 8 horas lectivas y con una actividad central, las "Estaciones de Aprendizaje". La asignatura de Antropología Social en este grado debe proporcionar a los estudiantes una formación universitaria orientada a promover la capacidad reflexiva y crítica para comprender las claves de los diferentes sistemas socioculturales, incluido el propio, favoreciendo, si es posible, el papel activo y emprendedor del alumnado. El objetivo de este ciclo de mejora fue convertir el proceso de enseñanza-aprendizaje en algo dinámico y participativo. Para su implementación se siguió una metodología basada en la investigación. Los resultados muestran valoraciones positivas que se reflejan en forma de escaleras de aprendizaje.

Palabras clave: Antropología Social; Grado en Criminología; Docencia universitaria; Experimentación docente universitaria; Estaciones de aprendizaje.

\section{Abstract}

In Social Anthropology subject, from the first year of the Degree in Criminology, an Improvement Cycle in Classroom (ICIC) was developed in the topic of the subject titled "Social and Political Organization", occupying the cycle a total of 8 teaching hours and with a central activity, the "Learning Stations". The subject of Social Anthropology in this degree should provide students with a university formation aimed at promoting reflective and critical capacity to understand the keys of different sociocultural systems, including their own, favoring, if possible, the active and entrepreneurial role of the students. The aim of this improvement cycle was to turn the teaching-learning process into something dynamic and participative. For its implementation, a research-based methodology was followed. The results show positive valuations that are reflected as learning ladders.

Keywords: Social Anthropology; Degree in Criminology; University teaching; University teaching experience; learning stations. 


\section{Contexto de la intervención}

El presente artículo tiene como finalidad la descripción de un Ciclo de Mejora en el Aula (CIMA) aplicado durante el curso académico 2018/19 en la asignatura Antropología Social que forma parte del plan de estudios del Grado en Criminología en la Universidad de Sevilla. Se trata de una asignatura troncal de carácter obligatorio que tiene una carga docente de 6 créditos ECTS, esto es, 60 horas presenciales de clases que se imparten durante el segundo cuatrimestre. En el curso donde se ha aplicado el ciclo de mejora docente hay matriculados un total de 62 estudiantes, 17 chicos y 45 chicas (17,4\% y 72,6\% respectivamente). Al tratarse de una asignatura del primer curso, mayoritariamente son alumnos y alumnas que comienzan su carrera universitaria.

La asignatura de Antropología Social, en el Grado de Criminología, ocupa cuatro horas semanales, de lunes a jueves, 60 minutos cada día. Esta materia está planificada a priori con dos tipos de clases. En primer lugar, clases teóricas en las que la profesora presenta el contenido teórico de la asignatura a través de presentaciones de power point y lecturas complementarias, y, en segundo lugar, clases prácticas en las cuales el alumnado realiza actividades prácticas y de desarrollo vinculadas al contenido teórico impartido. En lo que respecta a la evaluación de la asignatura, para la parte teórica se contempla la posibilidad de realizar una evaluación continua (a través de tres trabajos) o una evaluación final (examen oficial). La parte práctica es evaluada mediante la entrega de un portafolio que recopila las actividades realizadas a lo largo del curso.

Para el desarrollo de este ciclo se ha tenido en cuenta el conocimiento adquirido en el Curso General de Docencia Universitaria (CGDU), realizado por la docente, así como en lo aportado en las experiencias expuestas por Bain (2008) y Finkel (2008), que se han convertido en referencias de 
cabecera para la actuación en el ámbito de la innovación docente universitaria.

El CIMA que se llevó a cabo tuvo una duración de 8 horas lectivas y se implementó en la segunda parte del tema 3 , de un total de 5 temas que componen el marco teórico y conceptual de la materia. Este tema se titula "La organización social y política" y, de acuerdo con los objetivos generales del Grado en Criminología en el que se imparte, este ciclo de mejora está orientado por los siguientes objetivos:

1. Acercar a las alumnas y alumnos a una actitud crítica y de compromiso frente a los problemas sociales y culturales. Introducir al alumnado en los principales contenidos y conceptos de la organización social y política dentro de la Antropología social y cultural, especialmente en su relación con la Criminología.

2. Promover la capacidad reflexiva y crítica para comprender las claves de los diferentes sistemas socioculturales, incluido el propio.

3. Desvelar las bases sobre las que se construyen los discursos y prácticas racistas, xenófobos, sexistas, clasistas y excluyentes relacionados con la transmisión cultural de cada modelo de organización social.

Relacionados con estos objetivos se delimitan las siguientes competencias:

1. Poseer y comprender conocimientos actualizados de las principales instituciones de control social.

2. Manejar, desde la visión socio-antropológica, los conceptos de violencia, desigualdad y diferencia, delincuencia, control social, estigmatización, desviación social.

3. Aplicar conceptos y metodologías de redes sociales y de modelos de organización social a procesos de criminalidad. 


\section{Mapa de contenidos y modelo metodológico}

El contenido teórico del Tema 3, en el que se aplicó el ciclo de mejora fue el siguiente: Organización social y política. El parentesco. Tipos de familia, matrimonio y filiación. Poder y control social. Política, poder, autoridad y liderazgo. Formas de organización política: Bandas, tribus, jefaturas y estados. Debido a la organización temporal de la asignatura y del propio CIMA se excluyó de este la parte que hacía referencia al parentesco, los tipos de familia, el matrimonio y la filiación, que se desarrollaron en clases previas a la intervención.

A continuación, se presenta un mapa con los contenidos conceptuales que se trabajaron (Figura 1).

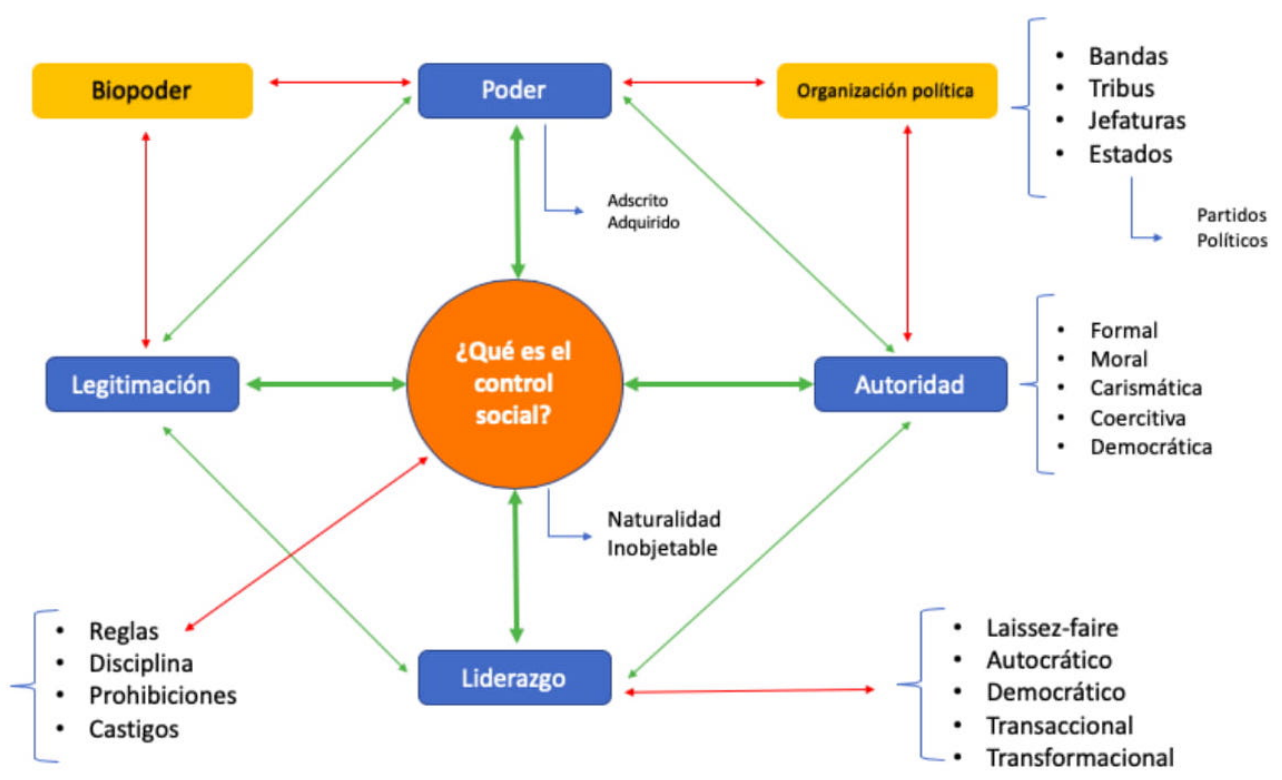

Concepto central

Conceptos principales

Conceptos secundarios

Figura 1. Mapa de contenidos conceptuales.

Jornadas de Formación e Innovación Docente del Profesorado | № 2 (2019) Esta obra se distribuye con la licencia Creative Commons Reconocimiento-NoComercial-SinObraDerivada 
En el mapa de contenidos vienen reflejados los contenidos conceptuales impartidos durante el CIMA tal y como se les presentó al alumnado. En cuanto a los contenidos procedimentales utilizados, se plantearon mediante la recopilación y sistematización de información, así como la práctica en diferentes formas de transmitir el conocimiento entre ellos mismos, aplicado a las actividades diseñadas. Los contenidos actitudinales, los componentes de comportamiento necesarios y observables en su interrelación con los iguales, fueron muy importantes. Estos guiaron el aprendizaje de los contenidos conceptuales y procedimentales y propiciaron la incorporación de valores en el alumnado, favoreciendo, en definitiva, su formación integral. Por ello, fue básico establecer el CIMA como una propuesta de trabajo cooperativo, solidaridario y de responsabilidad grupal.

En cuanto al modelo metodológico planteado, se contempló un modelo basado en el principio de investigación y reelaboración de ideas por parte de los estudiantes (De Alba y Porlán, 2017). Es decir, se partió de un problema (cuestión) formulado en este caso como proyecto sobre qué es el control social, sus características y con qué elementos se relaciona. Este planteamiento debía activar el interés, la implicación y respuestas del alumnado. Para ello se utilizarán diversos recursos y actividades, así como conocimiento ya manejado que se reactivó, concluyendo con la comparación de las ideas iniciales y finales de los estudiantes.

Como se indica en el apartado anterior, en esta asignatura, la metodología de enseñanza habitual consiste en clases transmisivas donde la docente posee el papel central y clases prácticas donde se intenta aplicar el contenido teórico a experiencias reales. Por tanto, se encuadra la metodología de enseñanza habitual en el modelo "basado en la teoría y su aplicación práctica” (De Alba y Porlán, 2017). Al pasar al modelo metodológico más complejo 
propuesto en el CIMA, se diseña el modelo representado en la Figura 2, que comenzó con el planteamiento inicial de una pregunta clave de formulación abierta, cuya respuesta se fue elaborando conforme avanzaron las diferentes actividades planteadas.

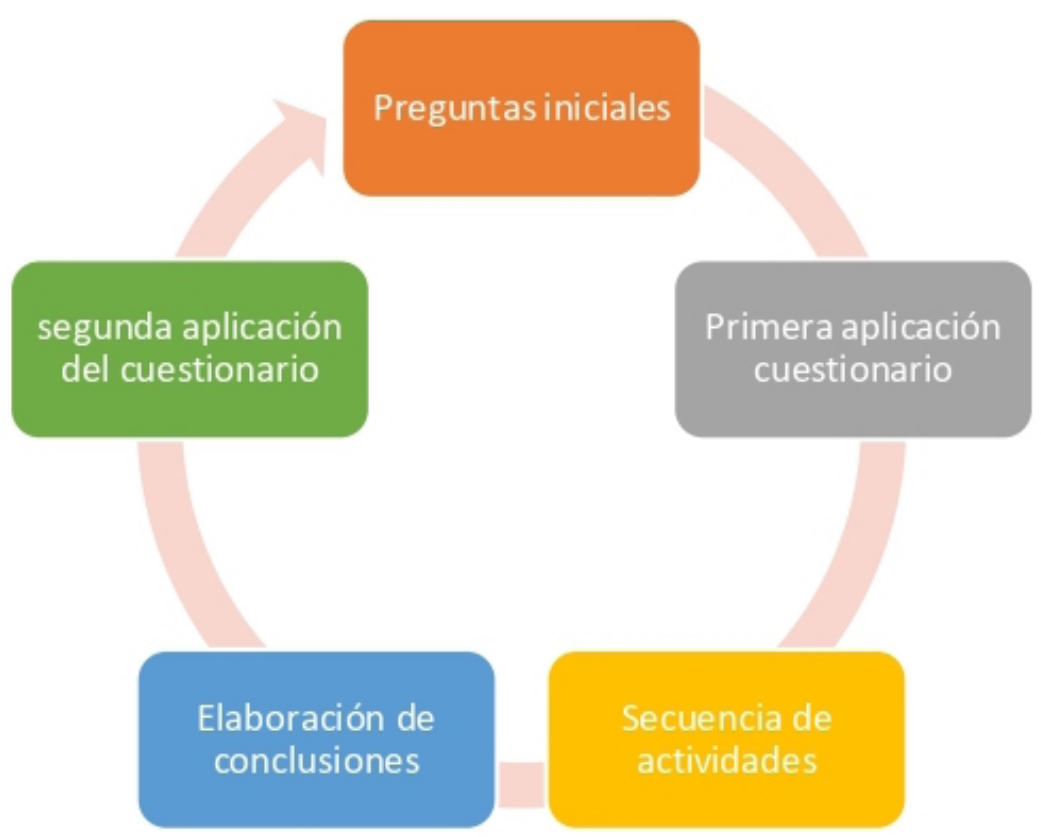

Figura 2. Modelo metodológico del Ciclo de Mejora en el Aula.

\section{Secuencia de actividades}

La secuencia de actividades llevada a cabo en este CIMA se diseñó en base a una actividad central, las "estaciones de aprendizaje". Así, se planificaron 6 actividades. La actividad 1 y la 6 , primera y última respectivamente, fueron los cuestionarios de evaluación. La actividad 2 se contempló como una clase teórica que sirviera de introducción para el resto de actividades. La actividad 3, las "estaciones de aprendizaje", fue la actividad más extensa de las planificadas. La actividad 4 se diseñó para introducir la perspectiva de género y la actividad 5 para que el 
alumnado pudiera relacionar de manera más evidente la Criminología con el contenido impartido.

Las "estaciones de aprendizaje" planteadas en el presente CIMA es una actividad inspirada en la metodología constructivista desarrollada por el profesor Bauer (2009). El objetivo principal de esta actividad, además de lograr el aprendizaje del alumnado es utilizar el aprendizaje por estaciones como una herramienta al alcance del docente para mejorar el proceso de enseñanza-aprendizaje en asignaturas especialmente relacionadas con las Ciencias Sociales como es la Antropología social. La idea principal de esta herramienta consiste en que el alumnado trabaje una parte del contenido teórico, repartido en diferentes estaciones de trabajo. Es decir, el docente reparte el contenido (epígrafes) que quiere trabajar en diferentes partes que quedan a disposición de los alumnos y alumnas en las estaciones repartidas por el aula. En lugar de ir introduciendo por parte del docente el material de forma progresiva, el alumnado trabajando por grupos de manera autónoma, siguiendo unas pequeñas instrucciones, construye el contenido teórico planteado. De esta manera, cada estación es un grupo de trabajo y el conjunto de todas las estaciones distribuidas en el aula puede denominarse "circuito de aprendizaje". El alumnado, una vez finalizado su trabajo de construcción del conocimiento dentro de su estación, "viaja" hasta la estación siguiente donde los representantes, previamente elegidos, de ese espacio les muestran todo lo aprendido (a través de un mural) produciéndose de esta manera un proceso de enseñanza-aprendizaje horizontal y colaborativo. Este proceso se da hasta que todo el alumnado haya "viajado" a cada una de las "estaciones de aprendizaje", al final del cual, los estudiantes tienen que haber logrado su propio conocimiento a través de su trabajo. Se trata de adquirir un objetivo de aprendizaje establecido a priori, paso a paso, o estación tras estación. El aprendizaje que deriva, por tanto, de esta actividad, potencia un desarrollo gradual de interacción entre 
las personas, así como la integración de todo el alumnado como parte importante de la dinámica de clase, con las consecuencias positivas que ello conlleva.

Las "estaciones de aprendizaje" y el resto de actividades planteadas en el CIMA se recogen en la Tabla 1, a continuación.

Tabla 1. Secuencia de actividades del CIMA.

A1: DESCRIPCIÓN DE LA ACTIVIDAD
Aplicación de cuestionario inicial.
Realización de un cuestionario inicial con 4 preguntas abiertas donde se
recogerá el conocimiento de partida sobre los contenidos teóricos tratados
en el ciclo de mejora.
SENTIDO EDUCATIVO
La aplicación de este cuestionario tiene como objetivo realizar una
evaluación inicial que sirva como punto de partida para la docente y como
referente para la evaluación final.
CONTENIDOS
IV. Cuestionario con cuatro preguntas:
¿Qué es el control social?, ¿qué función cumple?
¿Qué es la organización social y cómo se relaciona con el control social?
Pon algún ejemplo.
¿Cómo afecta el control social al delito? Pon algún ejemplo.

A2: DESCRIPCIÓN DE LA ACTIVIDAD

Teoría-debate. Introducción al control social.

Clase transmisiva sobre el concepto principal en el cual se imbrican todos los contenidos del tema que estamos tratando. Esta actividad se inicia con una pregunta: ¿Qué es el control social? Una vez ofrecido el contenido teórico el alumnado intentará relacionar el concepto presentado con la primera parte del tema, que habíamos visto en el primer ciclo de mejora (el parentesco, el matrimonio, la filiación y los tipos de familia). Otras preguntas planteadas y a las que se tratará de dar respuesta tras la exposición son: ¿Para qué sirve el control social? ¿Sabrías poner un ejemplo de control social informal? ¿En qué casos el control social formal es necesario? ¿Sabrías relacionar el control social y sus tipologías con la producción del delito? En todo momento se anima al alumnado a participar.

Jornadas de Formación e Innovación Docente del Profesorado I № 2 (2019) Esta obra se distribuye con la licencia Creative Commons Reconocimiento-NoComercial-SinObraDerivada Internacional (CC BY-NC-ND 4.0.) 


\section{SENTIDO EDUCATIVO}

Se trata de facilitarles a los alumnos a nivel teórico el concepto principal alrededor del cual se desarrollarán el resto de contenidos que vamos a trabajar en las siguientes actividades.

Se trata de anticipar conceptos teóricos haciéndolo desde el propio razonamiento y reflexión.

\section{CONTENIDOS}

Organización política: bandas, tribus, jefaturas y Estados.

A3: DESCRIPCIÓN DE LA ACTIVIDAD

Las "estaciones de aprendizaje".

Esta actividad está dividida en dos partes interrelacionadas entre sí.

Parte 1. Se divide la clase en cuatro grupos iguales; cada uno de esos grupos trabajará de manera independiente un modelo de organización social y política (tribus, clanes, jefaturas y Estados) y mostrará el contenido en dos cartulinas. Cada grupo se gestionará de manera autónoma para organizarse y repartirá las tareas.

La docente sólo da unas breves indicaciones procedimentales:

- El grupo de las bandas tendrá un líder que dirigirá el trabajo.

- El grupo de las tribus tendrá un líder y un supervisor; el supervisor en este caso es el único que se comunicará con el resto de componentes para darle instrucciones de qué tienen que hacer.

- El grupo de las jefaturas tienen un consejo formado por tres miembros, donde se tomarán las decisiones en relación al trabajo.

- El grupo del Estado se organizarán de manera horizontal, sin liderazgos. En cuanto a las indicaciones conceptuales, se ofrece una diapositiva con los elementos básicos que deben aparecer en el trabajo. Son estos:

1. Número de habitantes (aprox.)

2. Tipo de organización social (igualitaria, jerarquizada...)

3. Economía

4. Reglas de parentesco

5. Religiosidad

6. Otras características

Una vez que los grupos hayan terminado de construir el conocimiento sobre cada uno de los modelos de organización social y política que les ha tocado, comienzan la segunda parte de la actividad.

Jornadas de Formación e Innovación Docente del Profesorado | № 2 (2019) Esta obra se distribuye con la licencia Creative Commons Reconocimiento-NoComercial-SinObraDerivada Internacional (CC BY-NC-ND 4.0.) 
Parte 2. Cada grupo conforma una "estación de aprendizaje" que funcionará cuando otro grupo llegue a visitarlos. De manera que los grupos van rotando por cada una de las estaciones donde un representante les explicará en qué consiste su modelo de organización social y política.

Todos los grupos pasarán por todas las estaciones.

Al finalizar la actividad se realizará una asamblea reflexiva donde se comparta la concepción que cada uno ha tenido sobre esta experiencia de aprendizaje, que a su vez servirá de feedback para la docente.

\section{SENTIDO EDUCATIVO}

Se trata de que el alumnado sea sujeto activo del proceso de enseñanza aprendizaje. Construye el propio conocimiento y lo transmite al resto de compañeros/as. Actividad basada, por tanto, en el trabajo cooperativo donde el desarrollo de habilidades comunicativas, de colaboración y el conocimiento compartido son ejes fundamentales. Así como la autonomía y autogestión del contenido a tratar y a transmitir.

\section{CONTENIDOS}

Organización política: bandas, tribus, jefaturas y Estados.

\section{A4: DESCRIPCIÓN DE LA ACTIVIDAD}

El liderazgo y la autoridad.

La actividad se organiza en dos partes.

Parte 1. Exposición teórica sobre el liderazgo, qué es, los tipos de liderazgo y su relación con la estructura social a la que pertenecen.

Parte 2. Mix de actividades:

1. Confecciona una lista con tres personajes históricos que consideren líderes y responder a: ¿Qué tienen en común?

2. Indica en un máximo de tres líneas por qué son o fueron líderes y en qué campos las siguientes mujeres: Victoria Kent, Clara Campoamor, Rigoberta Menchú, Michelle Bachelet, Teresa de Calcuta.

\section{SENTIDO EDUCATIVO}

A través de diferentes actividades es posible lograr un aprendizaje significativo si el alumnado logra trasladar el contenido teórico impartido en la primera parte de la actividad a las diferentes actividades planteadas posteriormente.

\section{CONTENIDOS}

Autoridad y liderazgo, características y sus diferentes tipologías. 


\section{A5: DESCRIPCIÓN DE LA ACTIVIDAD}

\section{El biopoder}

Se trabajará en clase el capítulo "La corporación en crecimiento" correspondiente a la parte 2 del documental "La corporación" (Achbar et al., 2005). Una vez visto se plantea un debate donde se exponen los argumentos principales del documental y su relación con el concepto de control social visto hasta ahora.

Es importante que el alumnado sea capaz de identificar ejemplos cotidianos y reflexionar sobre ellos.

\section{SENTIDO EDUCATIVO}

Esta actividad se plantea desde dos dimensiones: por un lado fomenta el análisis crítico de un documental y la capacidad de sacar las ideas principales y aplicarlas a la realidad y por otro lado comprende el conocimiento teórico necesario para completar conceptualmente el tema "control social".

\section{CONTENIDOS}

Poder, biopoder, biopolítica.

A6: DESCRIPCIÓN DE LA ACTIVIDAD

Aplicación de cuestionario final.

Realización del mismo cuestionario que se hizo el primer día de la aplicación del ciclo de mejora donde se recogerá el conocimiento de final sobre los contenidos teórico/prácticos tratados.

\section{SENTIDO EDUCATIVO}

La aplicación de este cuestionario tiene como objetivo realizar una evaluación final que sirva como herramienta para el docente y recapitulación del conocimiento adquirido para el alumnado.

\section{CONTENIDOS}

Cuestionario con tres preguntas:

- ¿Qué es el control social y qué función cumple?

- ¿Qué es la organización social y cómo se relaciona con el control social? Pon algún ejemplo.

- ¿Cómo afecta el control social al delito? Pon algún ejemplo. 


\section{Aplicación del CIMA: relato resumido de las sesiones}

La herramienta del diario del profesor sin duda ha sido muy útil para ir anotando, de manera pormenorizada, las diversas situaciones que se dieron en cada una de las actividades que se han desarrollado en el CIMA.

\section{Actividad 1: Aplicación del cuestionario inicial. Resultados}

Comencé la clase explicando que iba a realizar un ciclo de mejora docente que consistía en la realización de una serie de actividades con un sentido bien definido y una finalidad previamente establecida. Les expliqué a los estudiantes que había elegido su curso porque consideraba que era un alumnado muy receptivo y los resultados alcanzados podían ser muy positivos para todos. Les entregué un folio con un cuestionario inicial formado por tres preguntas y les insistí en que no se iba a calificar. En el reverso del mismo documento vienen las mismas cuestiones, pero con el título de cuestionario final y les pedí que contestaran al inicial y que se quedaran con el documento.

\section{Actividad 2: Teoría-debate: Introducción al control social. Resultados}

La actividad consistió en una clase transmisiva introductoria al concepto de control social. Como en el primer ciclo de mejora habíamos trabajado la primera parte del tema (el parentesco, la familia y la filiación) intercalaba cuestiones que relacionan las dos partes con la intención de que el alumnado comprendiera la continuidad de los contenidos y estableciera relaciones. Algunas de estas cuestiones fueron: ¿Cómo se relaciona el control social con nuestro modelo de familia? O ¿qué función cumplen los vecinos? En este caso les hablé del cliché de la típica "vecina alcahueta" (sobre todo en los pueblos) y su relación con el control social informal; esto les resultó muy 
divertido y estuvieron contando algunas anécdotas a este respecto. Además, se lanzaron otra serie de cuestiones vinculadas a la parte nueva que estábamos viendo como: ¿En qué casos el control formal es necesario? Esta cuestión les hizo reflexionar sobre los tipos de control social, sus funciones y limitaciones.

\section{Actividad 3: Las estaciones de aprendizaje. Resultados}

La actividad 3, por su enfoque y por su extensión, estaba organizada para que fuera el eje central del ciclo de mejora docente. En primer lugar, dividí a la clase en cuatro grupos (bandas, tribus, jefaturas y Estados); para ello, pedí al alumnado que se pusiera en la zona de la pizarra y los distribuí aleatoriamente en los cuatro grupos. Repartí a los chicos y chicas de esta forma, evitando que fueran ellos los que directamente se agruparan; ello facilitó que personas que nunca trabajaban juntas lo hicieran, y rompió la dinámica de relaciones establecidas dentro del aula. En la Figura 3 se contempla la distribución de los grupos dentro del aula.

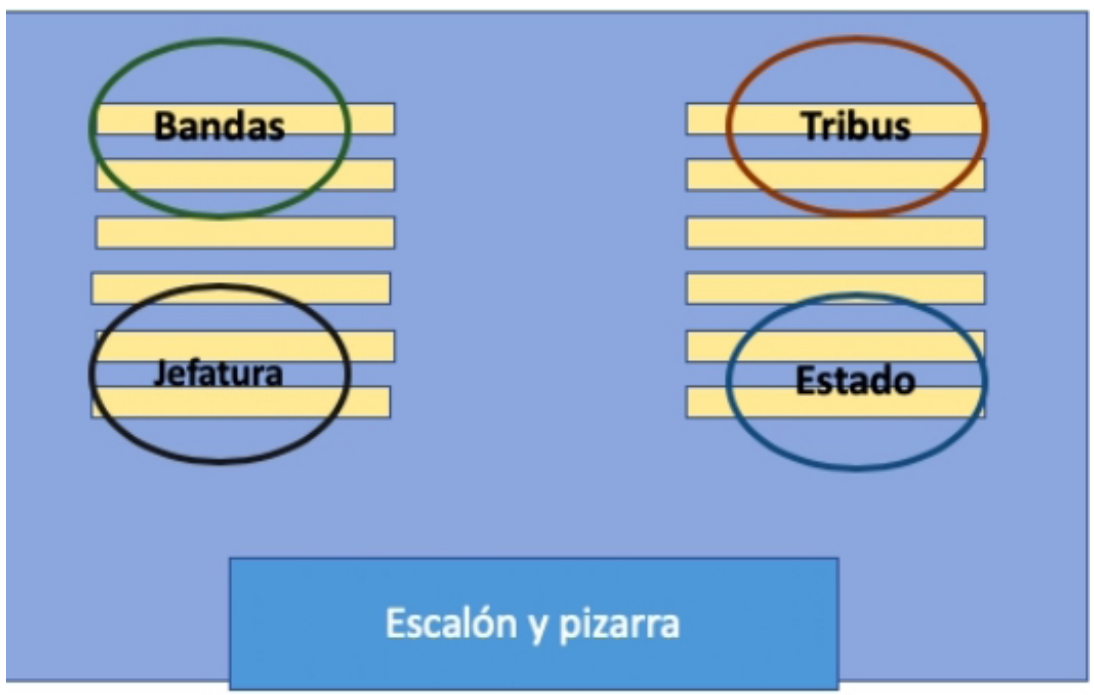

Figura 3. Disposición de las "estaciones de aprendizaje" dentro del aula.

Jornadas de Formación e Innovación Docente del Profesorado | № 2 (2019) Esta obra se distribuye con la licencia Creative Commons Reconocimiento-NoComercial-SinObraDerivada 
Una vez distribuidos por el aula les ofrecí dos cartulinas a cada grupo y les di las instrucciones. Deberían construir ellos mismos el conocimiento sobre la forma de organización política y social que les había correspondido. A lo largo del proceso me estuvieron preguntando sobre qué información seleccionar y les ofrecí una diapositiva con unos conceptos clave que debían aparecer en cada trabajo; esto les ayudó mucho. La realización de esta primera parte ocupó 2 horas. La segunda fase de las estaciones de aprendizaje consistió en la elección de 2 a 3 miembros en cada grupo que serían los que explicarían el contenido a los otros grupos y el resto rotaría en el sentido de las agujas del reloj hasta la siguiente estación. El tiempo aproximado de permanencia de los alumnos en cada estación era de 8 a 10 minutos. Cuando un grupo terminaba, aplaudían y se cambiaban todos los grupos hasta la nueva estación. Una vez finalizado el ciclo, rotaba el alumnado que había permanecido explicando en la estación y otros miembros ocupaban su lugar; esto se hizo para que ellos también realizaran el recorrido. La actividad fue un éxito, los estudiantes colaboraron, respetaron los tunos, se implicaron y, lo más importante, aprendieron. Posteriormente se eligió al grupo que mejor lo había hecho, evaluando tanto las cartulinas como la explicación de las mismas; en este caso ganó el grupo de las bandas. Lo que considero que no funcionó del todo bien, o es susceptible de volver a ser revisado, fueron las indicaciones que se ofrecieron sobre el tipo de autoridad que debía tener cada grupo para realizar el trabajo. Considero que supieron autogestionarse sin necesidad de llevar a cabo este elemento.

\section{Actividad 4: El liderazgo y la autoridad. Resultados}

Empecé la actividad con una exposición teórica sobre el liderazgo y la autoridad. Posteriormente se hicieron un conjunto de ejercicios, a modo de pasatiempos para trabajar de manera lúdica esta parte del contenido. En la primera tarea tenían que confeccionar una lista con tres 
personajes históricos que considerasen líderes y tenían que responder a la pregunta: ¿Qué tienen en común? Esta actividad les resultó agradable, hubo quien lo trabajó en grupo, otros de manera individual; el caso es que al final salieron mayoritariamente los mismos líderes históricamente más reconocidos (Gandhi, Che Guevara, madre Teresa de Calcuta, Jesús...). El hecho de que se llegara a esta conclusión me llevó a introducir la segunda tarea, buscar y escribir en tres líneas por qué fueron líderes 4 mujeres que les puse en la pizarra (descritas en el diseño del ciclo), mujeres prácticamente desconocidas para ellos. El introducir nuevos nombres y femeninos abrió un debate muy interesante sobre el reconocimiento de la mujer en el espacio público, la socialización de género etc., cuestiones que habíamos visto en el tema 2 de la asignatura y que ahora podíamos relacionar con algo aparentemente ajeno.

\section{Actividad 5: El biopoder. Resultados}

La parte del biopoder tenía especialmente ganas de impartirla porque es un tema que suele acarrear filias y fobias, muy visceral. La actividad estuvo compuesta por una primera parte de introducción teórica y una segunda parte con el visionado de un capítulo del documental La corporación (https://www.youtube.com/watch?v=Bkr-paaAY18\&t=4202s), concretamente el titulado "La corporación en crecimiento" -los niños como consumidores- y donde se muestra cómo empresas hacen estudios para saber cómo hacer que los niños se sientan felices con satisfacer necesidades creadas, consumiendo; para ello se les inculca determinados valores llegando al punto de convertirlos en seguidores de determinadas marcas desde pequeños. Hubo dos posturas principales durante el debate: los que, a pesar de la parte teórica y del contenido del documental, consideraban cierto grado de voluntariedad y los que desde el principio estaban de acuerdo en el control del cuerpo por parte de grandes corporaciones y sobre todo desde la biopolítica. Ciertamente el contenido mismo del debate me supuso una grata sorpresa porque cuando 
empezamos el curso (es alumnado de primero) no me imaginaba que pudieran alcanzar este nivel de reflexión.

\section{Actividad 6: Aplicación del cuestionario final. Resultados}

Como el cuestionario que había entregado en la situación inicial tenía impreso en el reverso el cuestionario final, compuesto por las mismas cuestiones, solicité al alumnado que lo había traído que lo realizara. Esto me ha facilitado que las personas que contestaron el cuestionario inicial fueran las mismas que en el final. Se recabaron un total de 25 cuestionarios que se analizan posteriormente. Les expliqué que era el broche a la experiencia de mejora docente y que era muy importante que contestasen con honestidad.

\section{Evaluación del aprendizaje}

\section{El cuestionario}

En el CIMA se utilizó en primer lugar un cuestionario para evaluar los contenidos de la temática "control social" que sirvió, en primer lugar, para adaptar el nivel de profundización y, en segundo lugar, una vez finalizados estos contenidos, para evaluar el grado de aprendizaje que se ha producido en el alumnado (Rivero y Porlán, 2017).

En este caso, se analizaron las diferencias entre las respuestas iniciales y las finales para valorar si se modifican los porcentajes en la escalera de aprendizaje establecida a priori (Figura 4). Las preguntas planteadas fueron:

1. ¿Qué es el control social y qué función cumple?

2. ¿Qué es la organización social y cómo se relaciona con el control social? Pon algún ejemplo.

3. ¿Cómo afecta el control social al delito? Pon algún ejemplo. 
No responde o lo hace de manera beve y simple.
Responde de manera descriptiva sin relacionar ideas ni utilizar un lenguaje específico. No pone ejemplos o estos son simples.
Aunque responde de forma descriptiva, usa un lenguaje específico. Los ejemplos son pertinentes.
Usa un lenguaje específico e interrelaciona ideas. Los ejemplos son complejos.

Figura 4. Escalera de evaluación del aprendizaje.

\section{Resultados}

El proceso de evaluación de los conocimientos iniciales y finales se realizó sobre un total de 25 estudiante matriculados. En la Figura 5 se observa la evolución de los resultados del conjunto de cuestiones de cada cuestionario. El escalón 1 formado inicialmente por el 32\% ocupó posteriormente el $2 \%$ del total. El escalón 2 compuesto por el $52 \%$ en el cuestionario inicial derivó en el final en el $33,3 \%$ del total. El escalón 3 formado por el $24 \%$ en el cuestionario inicial estuvo formado por el $49,4 \%$ en el cuestionario final. Y por último el escalón 4 que en el cuestionario inicial representaba el $0 \%$ del total creció hasta el 14,6\%. En general una evolución muy positiva.

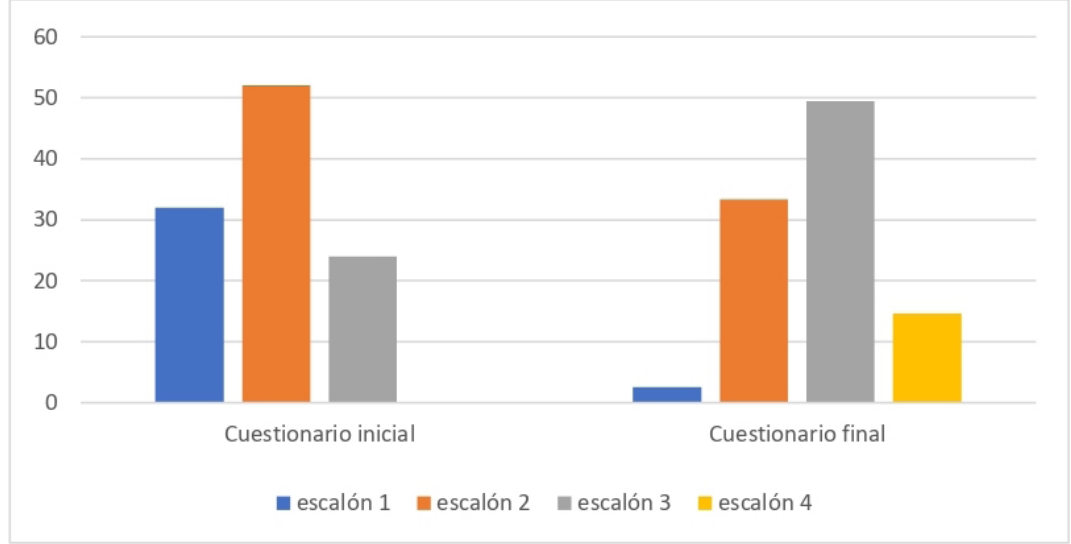

Figura 5: Evolución del alumnado según la escalera de aprendizaje.

Jornadas de Formación e Innovación Docente del Profesorado | № 2 (2019) Esta obra se distribuye con la licencia Creative Commons Reconocimiento-NoComercial-SinObraDerivada 
En la Tabla 2 se exponen los resultados comparativos del cuestionario inicial y final de manera individual. Como se puede apreciar, el $100 \%$ del alumnado que realizó el cuestionario inicial y el final sube de escalón en alguna de las tres preguntas. Específicamente, en la pregunta 1: ¿Qué es el control social y qué función cumple?, el 64\% del alumnado mejora los resultados con respecto al cuestionario inicial. En la pregunta 2: ¿Qué es la organización social y cómo se relaciona con el control social? Pon algún ejemplo, lo hace el 68\% del alumnado. Y finalmente, en la pregunta 3: ¿Cómo afecta el control social al delito? Pon algún ejemplo, cuestión que además relaciona directamente el contenido teórico con su titulación, mejora los resultados de nuevo el 68\% del alumnado que realizó las dos evaluaciones.

Tabla 2. Resultados individuales (indicando el escalón de la respuesta dada) de los cuestionarios inicial y final.

\begin{tabular}{|c|c|c|c|c|c|c|}
\hline \multirow{2}{*}{ ALUMNO/A } & \multicolumn{2}{|c|}{ PREGUNA 1 } & \multicolumn{2}{c|}{ PREGUNTA 2 } & \multicolumn{2}{c|}{ PREGUNTA 3 } \\
\hline & Inicial & Final & Inicial & Final & Inicial & Final \\
\hline 1. & 2 & 3 & 1 & 2 & 1 & 2 \\
\hline 2. & 2 & 2 & 2 & 2 & 1 & 2 \\
\hline 3. & 2 & 2 & 1 & 3 & 2 & 1 \\
\hline 4. & 2 & 2 & 2 & 2 & 2 & 1 \\
\hline 5. & 3 & 2 & 2 & 3 & 3 & 3 \\
\hline 6. & 2 & 2 & 3 & 3 & 3 & 2 \\
\hline 7. & 2 & 3 & 2 & 3 & 2 & 3 \\
\hline 8. & 2 & 2 & 2 & 2 & 1 & 2 \\
\hline 9. & 2 & 2 & 2 & 3 & 1 & 2 \\
\hline 10. & 2 & 3 & 3 & 3 & 2 & 3 \\
\hline 11. & 2 & 3 & 1 & 2 & 1 & 3 \\
\hline 12. & 2 & 3 & 2 & 3 & 2 & 4 \\
\hline 13. & 1 & 2 & 2 & 2 & 2 & 2 \\
\hline 14. & 2 & 4 & 2 & 3 & 3 & 3 \\
\hline 15. & 2 & 3 & 1 & 2 & 1 & 4 \\
\hline 16. & 1 & 3 & 1 & 2 & 2 & 2 \\
\hline 17. & 2 & 3 & 3 & 3 & 3 & 3 \\
\hline 18. & 3 & 4 & 3 & 4 & 2 & 3 \\
\hline 19. & 2 & 3 & 2 & 3 & 3 & 1 \\
\hline
\end{tabular}




\begin{tabular}{|l|l|l|l|l|l|l|}
\hline 20. & 3 & 3 & 2 & 3 & 2 & 3 \\
\hline 21. & 2 & 3 & 2 & 3 & 3 & 4 \\
\hline 22. & 2 & 4 & 1 & 3 & 1 & 4 \\
\hline 23. & 1 & 3 & 1 & 4 & 1 & 4 \\
\hline 24. & 1 & 3 & 1 & 3 & 1 & 2 \\
\hline 25. & 2 & 2 & 3 & 2 & 1 & 2 \\
\hline
\end{tabular}

\section{Conclusión. Modelo didáctico personal}

La participación en el Curso General de Docencia Universitaria y la implementación del CIMA me ha otorgado la posibilidad de definir un modelo didáctico propio que me ayude a enseñar buscando el enriquecimiento progresivo del conocimiento del alumnado hacia formas más complejas de entender su realidad (García Pérez y Porlán, 2017). Este modelo enseña desde un conocimiento que integra diversos referentes disciplinares y sobre todo cotidianos, para eliminar niveles de abstracción y favorecer el aprendizaje significativo. Todo esto gracias al planteamiento de una pregunta inicial que sea el hilo del que tirar para desenmarañar la madeja teórica.

En cuanto a cómo llevar a cabo todas estas cuestiones, considero que sin duda utilizando una metodología basada en la idea de investigación del estudiante (De Alba y Porlán, 2017). El trabajo se realiza en torno a problemas y con actividades diseñadas precisamente para la resolución de estos. Para ello, mi propuesta principal en el presente CIMA ha sido lo que he denominado "Estaciones de Aprendizaje". Con ellas, como contexto de investigación y de comunicación del conocimiento, el papel activo principalmente lo posee el alumnado como constructor de su propio proceso de aprendizaje. Y el papel del docente es el de acompañar, guiar el aprendizaje, asesorar pero también coordinar el mismo, aunque evidentemente sin perder la dimensión transmisiva en los momentos adecuados del proceso, pues es la base teórica que desde mi punto de vista resulta esencial plantear todavía en mis clases. 
En cuanto a la evaluación, esta experiencia me ha mostrado la importancia de realizar una evaluación inicial que complemente la evaluación sumativa utilizando la formativa si es preciso. Todas ellas necesarias para el seguimiento de la evolución del conocimiento del alumnado, sin olvidar la autoevaluación del docente.

Pero es cierto que todo este novedoso planteamiento didáctico, para mí como profesora, se convierte en un proyecto a modificar a largo plazo porque no sólo habría que modificar el paradigma docente sino también el que posee el propio alumnado como sujetos pasivos en el proceso de enseñanza-aprendizaje que viene dado por el sistema educativo vigente.

Jornadas de Formación e Innovación Docente del Profesorado | № 2 (2019) Esta obra se distribuye con la licencia Creative Commons 


\section{Referencias bibliográficas}

Achbar, A. (productor), Abbott, J., Achbar, M. y Bakan, J. (directores) (2005). The corporation [documental]. Canadá: Big Picture Media Corporation.

Bain, K. (2004). Lo que hacen los mejores profesores universitarios. Valencia: Publicacions de la Universitat de València.

Bauer, R. (2009). Lernen an Stationen weiterentwickeln. Wege zur Differenzierung und zum individuellen Lernen. Berlin: Cornelsen Verlag Scriptor GmbH \& Co.

De Alba, N. y Porlán, R. (2017). La metodología de enseñanza. En R. Porlán (Coord.), Enseñanza Universitaria. Cómo mejorarla (pp. 37-54). Madrid: Morata.

Finkel, D. (2008). Dar clase con la boca cerrada. Valencia: Publicacions de la Universitat de València.

García Pérez, F. F. y Porlán, R. (2017). Los Principios Didácticos y el Modelo Didáctico Personal. En R. Porlán (Coord.), Enseñanza Universitaria. Cómo mejorarla (pp. 93-104). Madrid: Morata.

Rivero, A. y Porlán, R. (2017). La evaluación de la enseñanza universitaria. En R. Porlán (Coord.), Enseñanza Universitaria. Cómo mejorarla (pp. 73-92). Madrid: Morata.

Jornadas de Formación e Innovación Docente del Profesorado | № 2 (2019) Esta obra se distribuye con la licencia Creative Commons 